\title{
REFLECTOR SURFACE MODELLING - A EUROPEAN COLLABORATION
}

\author{
Matteo Albani $^{(1)}$, Peter Balling ${ }^{(2)}$, Mauro Ettorre ${ }^{(3)}$, Giampiero Gerini ${ }^{(4)}$, Stefano Maci ${ }^{(5)}$, Knud Pontoppidan ${ }^{(6)}$, \\ Zvonimir Sipus $^{(7)}$, Daniel Sjöberg ${ }^{(8)}$, Giuseppe Vecchi ${ }^{(9)}$, Francesca Vipiana ${ }^{(10)}$ \\ ${ }^{(1)}$ University of Siena, Siena, Italy, Email: albani@dii.unisi.it \\ ${ }^{(2)}$ ASC, Selsmosevej 12, DK-2630 Taastrup, Denmark, Email: pballing@asc-consult.dk \\ ${ }^{(3)}$ University of Siena, Siena, Italy, Email: ettorre@dii.unisi.it \\ ${ }^{(4)}$ TNO, Oude Waalsdorperweg 63, The Hague, The Netherlands, Email: gerini@fel.tno.nl \\ ${ }^{(5)}$ University of Siena, Siena, Italy,Email: macis@dii.unisi.it \\ ${ }^{(6)}$ TICRA, Laederstraede 34,DK-1201 Copenhagen K, Denmark,Email: kp@ticra.com \\ ${ }^{(7)}$ University of Zagreb, Unska 3,Zagreb, Croatia, Email: zvonko.sipus@fer.hr \\ ${ }^{(8)}$ University of Lund, , Lund, Sweden, Email: daniel.sjoberg@es.lth.se \\ ${ }^{(9)}$ Politecnico di Torino, Torino, Italy, Email: giuseppe.vecchi@polito.it \\ ${ }^{(10)}$ Politecnico di Torino, Torino, Italy, Email: francesca.vipiana@polito.it
}

\begin{abstract}
The topic of this paper is the work carried out in Work Package 2.3-2 of the EU network ACE. This work package is concerned with the modelling of the surfaces of modern reflector antennas. In particular the problems associated with homogenisation of periodic structures are described together with an application example. An accurate MoM solutions for periodic structure is presented. A new, fast and very efficient interpolation technique for frequency selective surfaces is introduced and linked to high frequency techniques to speed up radiation calculation processes.
\end{abstract}

\section{INTRODUCTION}

In this paper, the work carried out in Work Package 2.32 of the EU network ACE, Antenna Centre of Excellence is described. This work package deals with the modelling of the surfaces of reflector antennas. Reflector antennas may operate over very wide bands typically limited by the feed system. Special surfaces are used either for polarization control or for frequency diplexing, using gridded surfaces or frequency selective surfaces, respectively. It may also be necessary to be able to take into account special surface elements such as Carbon Fibre Reinforced Plastics (CFRP), diffent types of mesh or paints. Reflector antennas find use in radio-links and, as antennas for geostationary satellites, both on ground as terminals and onboard the satellite. Alternatively special surfaces are used as radomes, to protect antennas under adverse climatic or environmental conditions, and on vehicles, trains aircraft, or ships, to reduce antenna aerodynamic profile or radar signature.

The list of authors shows the partners involved in this work package. Different topics are investigated by different partners as described in the following sections. It is important to stress, however, that the main purpose of the project was to combine the expertise available internally at each partner into a common and useful knowledge base.

\section{HOMOGENISATION}

Homogenization technique is usually employed to study problems where the operating wavelength is much larger than the microstructure scale. The idea is to replace a heterogeneous structure built up of small details with a effective, homogeneous structure, which would produce the same scattering characteristics. Strictly speaking, this can only be done in the limit where the wavelength is infinitely large compared to the microscopic details, but in actual engineering problems the homogenisation procedure still produces acceptable results for finite wavelengths. However, it is usually very difficult to deduce the range of validity for the homogenised results. Two separate questions are of particular interest:

1) Under which circumstances is it possible to model a particular structure as a homogeneous material? For most structures there is in general an infinite number of degrees of freedom for the electromagnetic field, corresponding to the number of modal solutions. By choosing the frequency low enough, the number of modes can be reduced to only correspond to the possible polarizations of the electromagnetic field.

2) Once the structure is modelled as a material, we may ask how strong the dependence on the scale difference between the wavelength and the microscopic structure is. This corresponds to identify the spatial dispersion, which is given by the dependence of the effective permittivity on $k a$, where $k$ is the wavenumber of the applied field and $a$ is the typical size of the microstructure. 
The main conclusion from the work carried out is that a heterogeneous structure may be modelled as a homogeneous material, even if the applied wavelength is not infinitely large compared to the microscopic scale. A sufficient condition for arbitrary geometries has been determined, but a wider range of validity for specific geometries can be anticipated.

As an example, the rigorous analysis of periodic strips inside a multilayer structure, illuminated by a plane wave, is performed by expanding the currents on the strips in basis functions, and the amplitudes of the basis functions are determined numerically by the Method of Moments (MoM). The electromagnetic field is in the form of Floquet modes due to the periodicity of the structure. It is sufficient to determine the current on one strip, since the currents on the other strips are identical except for a phase difference.

If the source excites a full spectrum of plane waves, such as a dipole, the Floquet-mode expansion/MoM is a laborious process. A simpler approach is to use approximate boundary conditions. We have used two types of approximate boundary conditions: the asymptotic strip boundary conditions (ASBC), which in the planar case correspond to model the strips as a unidirectional conducting screen, and boundary conditions obtained by the homogenisation method (BCHM).

The ASBC are applied to the components of the electromagnetic field that are tangential to the interface which contains the strips. The electric field component parallel to the strips is zero at the strip surface, and the component orthogonal to the strips is continuous across the surface. For the magnetic field it is sufficient to consider the component parallel to the strips, which is continuous across the surface.

The BCHM are obtained by averaging the fields of the fundamental Floquet mode. The zero-order boundary conditions correspond to ASBC, and the first-order boundary conditions include the periodicity, $P$, and the width, $W$, of the strips shown in Fig. 1.

As an example, Fig. 2 shows the reflection coefficient for a planar strip grid for different angles of incidence and as a function of the number of strips per wavelength. The polarisation of the incident plane wave is parallel to the strips. The BCHM results are compared to the MoM solution and an almost perfect agreement is obtained.

\section{FSS LARGE REFLECTORS}

Sheet polarisers typically consist of a number of thin layers with strips or some type of frequency selective surface (FSS). For the analysis of such reflectors it is essential that fast and reliable software is available to model the local reflection and transmission properties everywhere on the surface.

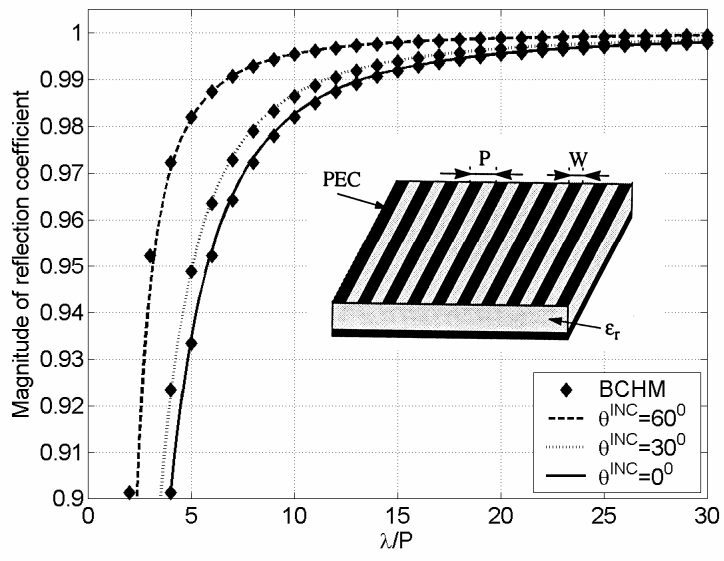

Figure 2. MoM (continuous curves) and BCHM results for the absolute value of the reflection coefficient of a planar strip grid for transverse incidence for $W / P=0.25$.

\subsection{FSS Integral equation solver}

The Politecnico di Torino and the University of Siena developed a code based on an efficient hybrid spatialspectral selective surface solver $(5 \mathrm{~S})$, for the analysis and design of planar periodic structures embedded in a multilayer substrate. The code has been developed under financial support of ESA-ESTEC under the contract Electromagnetic Antenna Modelling Library and coordinated in the ACE activity. The code is essentially a spatial domain code with phase sift boundary conditions, where the metallization in the periodic cell is discretized by a triangular mesh on which RWG basis functions are defined [1]. The periodic lattice can be rectangular or skew. Arbitrary stratification and various metallizations levels can be set-up. This code extends the previous spectral domain MESTIS code [2], that was limited to geometries consisting of a collection of slender strips, to the analysis of arbitrary shape geometries.

In 5S, the space domain periodic Green's function (PGF) is treated by extracting from the Floquet mode (FM) summation the summation of the dominant quasi static contributions expressed in terms of FMs. The residual series is converted with a simple mathematical trick into a rapidly convergent series of complex sources [3], [4]. This PGF is evaluated in a set of points inside the unit cell, and then it is interpolated to generate the MoM matrix of the unit cell. Finally, the problem relevant to the unit cell is solved and the scattering matrix of the periodic structure is evaluated.

The 5S code has been tested on several periodic structures in free space and in dielectric substrates and compared with commercial codes. 


\subsection{Fast interpolation for FSS}

Due to the large dimensions of the FSS, the asymptotic analysis is usually performed by resorting to highfrequency techniques, such as Physical Optics (PO) or Geometrical Optics (GO), sometimes augmented by diffraction theories (PTD, UTD, ITD). In this framework, it is desirable to have a simple and accurate surface impedance model of periodic surfaces, to be interfaced with existing high-frequency electromagnetic simulation tools. This model is extracted from the dominant FM of the exact MoM representation. Hower, the call on flight of the MoM code for each angle of local ray-incident field is a time consuming process not suitable for synthesis.

The idea is then to combine the accuracy of a rigorous solution with the speed of a fast interpolation technique. To this end, a method called the Pole-Zero Matching technique (PZM) has been developed, based on the location of the frequency domain singularities of the admittance matrix of the exact dominant FM.

The overall algorithm follows the flow chart of Fig. 3. On the basis of the $5 \mathrm{~S}$ code, an equivalent networkmatrix is defined with the ports corresponding to the accessible TE and TM FM of the exact Floquet expansion. The scattering matrix entries can be parameterized in terms of fractional polynomial, on the basis of the equivalent network interpretation. A compact analytical closed form expression, involving only few parameters to be computed, is thus obtained, which can be applied to evaluate the generalized scattering matrix as a function of the angle and polarization of the incident plane wave.

Though the full-wave analysis of $5 \mathrm{~S}$ is very efficient, the accurate calculation of the scattering matrix on the entire incidence direction $(\theta, \phi)$ domain, for many frequency points, requires a large amount of computational time. Therefore, the PZM method offers the possibility of reconstructing by an analytical expressions the generalized scattering matrix as a function of the incidence angles and frequency, starting from a rough sampling in angle. This is particularly useful to establish a link between an FSS solver and a $\mathrm{HF}$ solver for the analysis of a large FSS curved structure or frequency selective radome without affecting the internal code of the structure solver. Fig.3 shows as the PZM method can be used to link the code 5S and GRASP [5] without affecting the GRASP code and reducing the amount of information needed to recover the $5 \mathrm{~S}$ data. The general process described here can be applied for the synthetic description of different wave phenomena, like those relevant to surface wave propagation and electromagnetic band-gap description, near-field interaction (Green's function) and wave diffraction involving periodic surfaces.

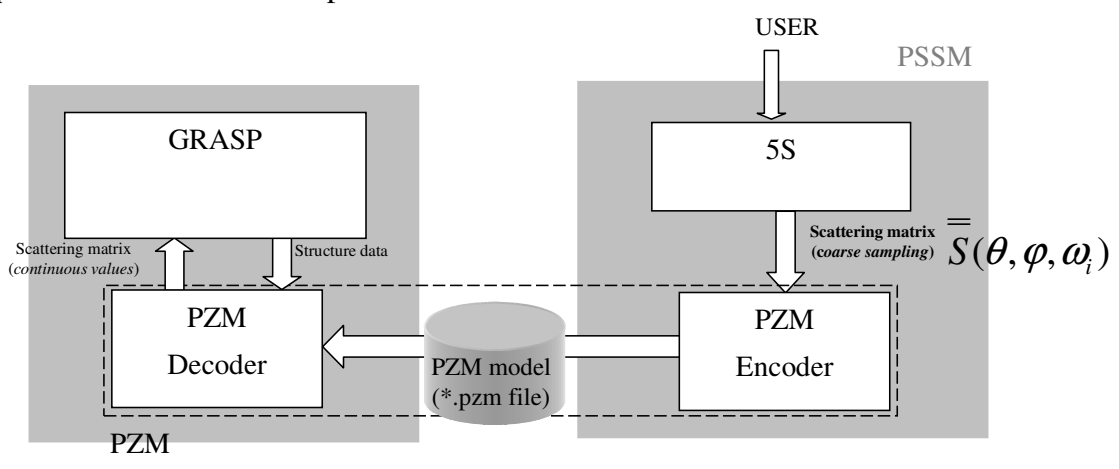

Figure 3. PZM method flow chart

\section{NEW ASYMPTOTIC METHODS FOR LARGE REFLECTORS}

To speed-up the radiation from large reflector, a formulation has been recently introduced to represent the PO scattering by a curved surface, in terms of GO contribution plus a line integration of incremental PO (IPO) contributions along the shadow boundary contour of the illuminated surface. This formulation, developed by the University of Siena in the framework of an ESTEC contract, named Shadow Boundary Integral (SBI) method, fits well both a Physical Theory of Diffraction (PTD) and the Incremental Theory of
Diffraction (ITD) scheme when corrected by the fringe contributions, and lies the basis to formulate ITD diffraction contributions that directly compensate through line integration the GO disappearance without resorting to a "fringe" scheme (Fig 5).

The SBI method can used to model perfectly conducting surfaces, or in connection to the PZM, to analyze FSS, polarizers and dichroic reflectors. Such structures can be described by an equivalent impedance model, used to derive local PO currents associated to the dominant Floquet mode of the canonical planar tangent surface. 
The main improvement of the new SBI developed by University of Siena is concerned with the possibility to model the scattering from a large curved reflector in terms of a line integration plus GO also for anastigmatic incident and reflected rays. For curved surfaces this leads to introducing a correction additional term depending in analytical simple fashion upon the curvature radius at the incremental point. The accuracy, with respect to PO is excellent, except close to caustic, where the formulation suffers of the same impairment of ray-theories. However, the new developed formulation, which is only slightly time consuming with respect to UTD (but considerably faster than PO) is more accurate than conventional UTD when vertex contribution are present. An example relevant to a curved parabolic truncated-cylinder reflector is shown in Fig. 4, where the UTD is also shown by comparison; the latter fails wrt the SBI, which is indeed accurate everywhere, except close to caustic.

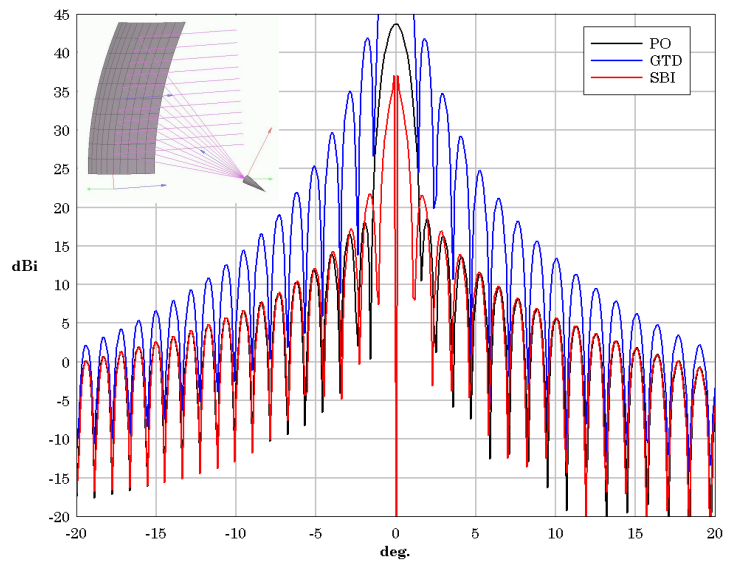

Fig.4. Comparison of $P O$ and SBI $D / \lambda=50$ Foci distance $=75 / \lambda$ Vertex distance $=25 / \lambda$ Gaussian type feed with $-12 \mathrm{~dB}$ taper at $25^{\circ}$ (edge illumination).

In order to overcome the problem of caustics, and to further widening the applicability of the method, the University of Siena has developed an extension to complex point sources of the SBI. The complex point sources behave paraxially like beams and serve to represent general incident fields. Till now, the extension of the SBI has been carried out for simple flat surfaces; results are shown in Fig. 5, with accuracy checked against MoM. The SBI method in those example, incorporate the correction provided by the Incremental Thory of Diffraction, too.

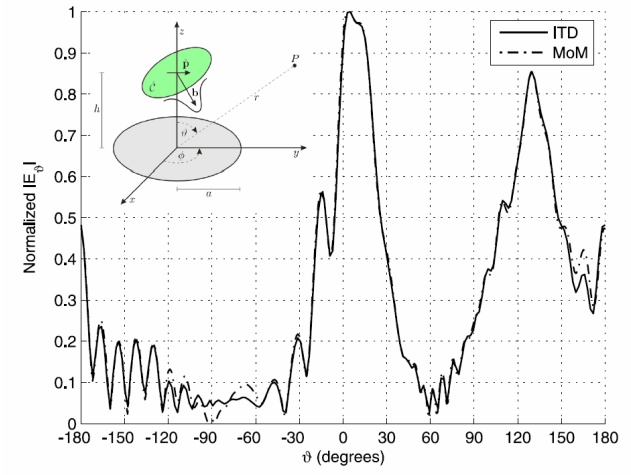

(a)

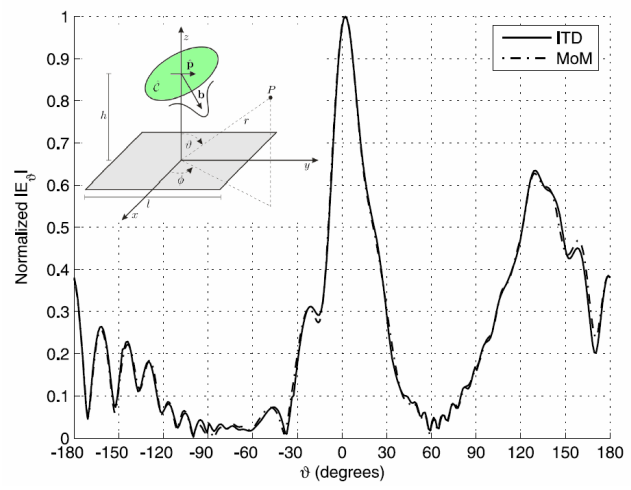

(b)

Fig. 5 Normalized electric field component of the field scattered by a flat plate illuminated by a complex point source. Comparison between IGO - ITD and MoM solutions. a) circular plate b) rectangular plate

\section{FUTURE ACTIVITY}

Current $\mathrm{C}-$, $\mathrm{Ku}-$ and Ka-band communications and broadcast satellites often use so-called dual-gridded reflector antennas for linear polarisation to provide independent reflector surfaces and/or independent feed arrays for the two orthogonal polarisations. In the case of shaped reflectors with single feeds, the beams in the two polarisations can be independently shaped. In the case of array-fed reflectors, the separate feed arrays for the two orthogonal polarisations simplify the feed-array layout and no orthomode transducers are required. It is of interest to extend these capabilities to circular polarisation, and it is also of interest to provide a polarisation reconfiguration capability so that the antenna may be switched providing either dual linear polarisation or dual circular polarisation, or the linear polarisation direction may be rotated. 
Obviously a polarisation switching or rotating capability may be incorporated in the array elements or feeds (illuminating a solid reflector). But for a large array, it is attractive to replace a large number of discrete expensive feed components by a single sheet polariser typically consisting of a number of thin layers with strips, meander lines or some other frequency selective surface. The space between the layers are often filled by lightweight foam or honeycomb about $\lambda / 4$ thick providing the necessary support and stiffness. Such structures may also play other roles, e.g. frequency diplexing and multiplexing, filtering etc.

The knowledge gained during the present work package forms the basis to study and prepare further developments of "dual-gridded" reflector antenna systems for circular polarisation with and without a capability for switching between dual linear and dual circular polarisation operation as well as other polarisation flexibility means.

\section{CONCLUSIONS}

This paper has briefly presented some of the topics that have been studied in Work Package 2.3-2 of ACE. A more detailed description of the work can be found in the report ACE-A2.3D4, which is available at the ACE web site on the internet [6].

\section{ACKNOWLEDGEMENTS}

The presents work is performed in a cooperation with the Antenna Center of Excellence (ACE). The activity presented in Sections 3 and 4 has been coordinated with the project EAML (Electromagnetic Antenna Modelling Library), financed by the European Space Agency (ESA-ESTEC, the Netherlands).

\section{REFERENCES}

[1] S.M. Rao, D.R. Wilton and A.W. Glisson, "Electromagnetic scattering by surfaces of arbitrary shape", IEEE Trans. Antennas and Propagat., vol. AP-30, n. 3, pp. 409-418, May 1982.

[2] R.Orta, R.Tascone and D.Trinchero, User Manual, Metallic Strips Simulator, Politecnico di Torino, Ref.: 012007/EM/RT, July 2001.

[3] F. Vipiana, A. Polemi, G. Vecchi, and S. Maci," Hybrid spatial-spectral analysis of periodic structures.", Antennas and Propagation Society International Symposium, pages 2867-2870. IEEE, 2006.
[4] R.M.Shubair, Y.L.Chow, "Efficient Computation of the Periodic Greens Function in Layered Dielectric Media", IEEE Transaction on Microwave Theory and Techniques, Vol. 41, no.3, March 1993, pp.498-502.

[5] K.Pontoppidan, GRASP9 Technical Description, TICRA Engineering Consultants, February 2005.

[6] http://www.antennasvce.org/ 